\title{
Compound heterozygous mutations of TYMP as underlying causes of mitochondrial neurogastrointestinal encephalomyopathy (MNGIE)
}

\author{
BUM CHUN SUH ${ }^{1}$, HA-NEUL JEONG ${ }^{1}$, BYUNG SUK YOON ${ }^{1}$, JI HOON PARK ${ }^{2}$, HYE JIN KIM ${ }^{2}$, \\ SUN WHA PARK ${ }^{3}$, JUNG HEE HWANG ${ }^{3}$, BYUNG-OK $\mathrm{CHOI}^{4}$ and $\mathrm{KI}^{\mathrm{WHA} \mathrm{CHUNG}}{ }^{2}$ \\ ${ }^{1}$ Department of Neurology, Kangbuk Samsung Hospital, Sungkyunkwan University School of Medicine, Seoul; \\ ${ }^{2}$ Department of Biological Science, Kongju National University, Gongju; ${ }^{3}$ DNA Analysis Division, National Forensic Service; \\ ${ }^{4}$ Department of Neurology, Ewha Womans University School of Medicine, Seoul, Republic of Korea
}

Received December 26, 2012; Accepted April 4, 2013

DOI: $10.3892 / \mathrm{mmr} .2013 .1479$

\begin{abstract}
Mitochondrial neurogastrointestinal encephalomyopathy (MNGIE), an autosomal recessive multiorgan disease, frequently associated with mutations in the thymidine phosphorylase (TYMP) gene. TYMP encodes thymidine phosphorylase (TP), which has an essential role in the nucleotide salvage pathway for mitochondrial DNA (mtDNA) replication. This study reports an MNGIE patient with novel compound heterozygous missense mutations (Thr151Pro and Leu270Pro) in TYMP. Each mutation was inherited from one parent. Neither mutation was found in the controls and the mutation sites were well conserved between different species. Neither large deletion nor causative point mutations were found in the mtDNA. The patient presented with MNGIE symptoms, including gastrointestinal discomfort, external ophthalmoplegia, pigmentary retinopathy and demyelinating type diffuse sensory motor polyneuropathy. The patient demonstrated an early-onset but mild phenotype, with 9.6\% TP activity; therefore, patients with these compound heterozygous mutations may exhibit a mild phenotype with a variable onset age according to TP activity level.
\end{abstract}

Correspondence to: Dr Ki Wha Chung, Department of Biological Science, Kongju National University, 182 Sinkwan-dong, Gongju 314-702, Republic of Korea

E-mail: kwchung@kongju.ac.kr

Dr Byung-Ok Choi, Department of Neurology, Ewha Womans University School of Medicine, Mokdong Hospital, 911-1 Mokdong, Seoul 158-710, Republic of Korea

E-mail: bochoi@ewha.ac.kr

Key words: Korean, mitochondrial DNA, mitochondrial neurogastrointestinal encephalomyopathy, mutation, TYMP

\section{Introduction}

Mitochondrial DNA depletion syndrome (MTDPS) consists of a group of genetically and clinically heterogeneous disorders caused by nuclear-mitochondrial intergenomic defects characterized by a significant reduction in mtDNA content (1-4). MTDPS is divided into several types (from MTDPS1 to MTDPS10) according to the distinct clinical phenotypes and genetic causes. The clinical phenotypes frequently overlap with each other, and thus require an exact diagnosis in order to distinguish each type. Mitochondrial neurogastrointestinal encephalomyopathy (MNGIE), a frequently diagnosed type of MTDPS, is an autosomal recessive multiorgan disorder characterized by gastrointestinal (GI) dysmotility, cachexia, myopathy, peripheral neuropathy, leukoencephalopathy and mitochondrial dysfunction. To date, three subtypes of MNGIE have been described; MTDPS1 (MIM\# 603041) caused by the thymidine phosphorylase (TYMP) gene on 22q13.33 (5), MTDPS4B (MIM\# 613662) caused by DNA polymerase $\gamma$ (POLG) on 15q26.1 (6,7) and MTDPS8B (MIM\# 612075) caused by $p 53$-inducible ribonucleotide reductase small subunit $($ RRM $2 B)$ on $8 \mathrm{q} 22.3(8,9)$. Mutations in POLG and RRM2B also cause MTDPS4A (Alpers' type) and autosomal-dominant progressive external ophthalmoplegia, respectively $(10,11)$.

Several homozygous or compound heterozygous mutations in TYMP have been reported to be the underlying causes of MNGIE (5,10-17). Thymidine phosphorylase (TP) encoded by $T Y M P$ catalyzes the phosphorylation of thymidine or deoxyuridine to thymine or uracil in mtDNA synthesis (4). The protein was originally identified as a platelet-derived endothelial cell growth factor (PDECGF), an angiogenic factor distinct from the previously described endothelial cell mitogens of the fibroblast growth factor family (18). A TP deficiency is a severe clinical condition in the affected tissues and is ultimately fatal (19). In a previous study, the inhibition of TP activity led to elevated pyrimidine levels and consequential axonal swelling (20). The inhibition of TP activity in TYMP knockout mice has been shown to result in the depletion or deletion of mtDNA $(4,21)$. Therefore, mutations in TYMP 
are relevant to the impaired replication and maintenance of mtDNA. On average, the onset of MNGIE in patients with a homozygous TYMP mutation occurs in the late teens, with death occurring in the thirties. However, patients with compound heterozygous TYMP mutations showed incomplete MNGIE phenotypes with a late onset occurring in the forties or fifties (15-17).

We identified a female Korean MNGIE patient with an early-onset but mild phenotype. The patient revealed typical MNGIE phenotypes, including GI discomfort, external ophthalmoplegia, pigmentary retinopathy and sensory motor polyneuropathy. Molecular genetic analysis revealed novel compound heterozygous mutations in TYMP, indicating that these particular mutations may be the genetic causes of the MNGIE phenotype.

\section{Materials and methods}

Subjects. A Korean MNGIE family (family ID: MT119) was enrolled in this study (Fig. 1). Furthermore, 225 healthy controls were recruited from the Neurological Department, Ewha Womans University, Mokdong Hospital (Seoul, Korea). The paternity of the patient was confirmed by genotyping 15 microsatellite markers using a PowerPlex 16 kit (Promega, Madison, WI, USA). This study was approved by the Institutional Review Board for Ewha Womans University, Mokdong Hospital and all participants provided written informed consent.

Clinical and electrophysiological assessments. Clinical information was obtained by neurological examination, including the assessment of muscle weakness, sensory impairment and reflexes. Muscle strength was assessed manually using the standard Medical Research Council (MRC) scale (http:// www.mrc.ac.uk). Nerve conduction studies of the median, ulnar, fibular, tibial, sural and superficial fibular nerves were determined. Electromyography (EMG) was performed in the first dorsal interosseous, biceps brachii, tibialis anterior, medial gastrocnemius and vastus lateralis muscles. Ultrasound examination for the peripheral nerves was performed on the bilateral median, ulnar, radial, sciatic, fibular, tibial and sural nerves. Blood TP activity was determined spectrophotometrically by measuring the conversion of thymidine to thymine in an end-point assay (Laboratory of Personalized Genomic Medicine, Columbia University, New York, NY, USA).

MRI studies. Whole brain MRIs were obtained from 5-mmslices without interslice gaps to produce 30 axial images using a 3.0-T system (Achieva, Philips, Netherlands). The imaging protocol consisted of T2-weighted spin echo (TR/TE=2,500/80 msec), T1-weighted spin echo $(\mathrm{TR} / \mathrm{TE}=400 / 10 \mathrm{msec})$ and fluid-attenuated inversion recovery (FLAIR; TR/TE=6,000/120 msec; inversion time, 2,000 msec) images.

Molecular study. Total DNA was extracted from leukocytes using a QIAamp Blood DNA kit (Qiagen, Hilden, Germany). Entire mtDNA was amplified by PCR using 46 primer sets from the MitoSEQr resequencing system (Applied Biosystems, Foster City, CA, USA). All coding exons of the PEO1, TYMP, ANT1, POLG1, POLG2, DGUOK,

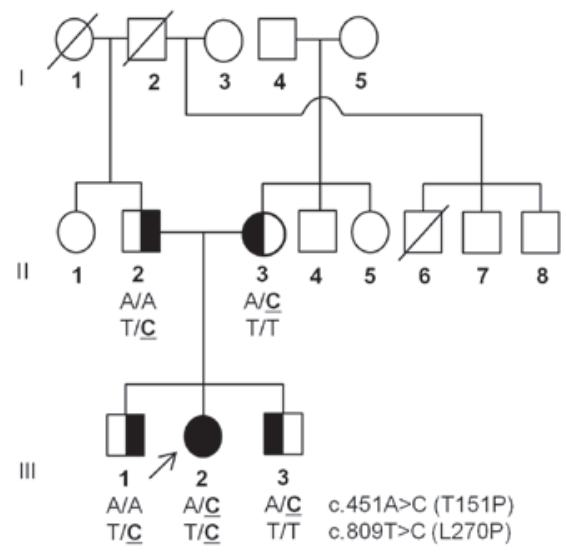

Figure 1. Pedigree of a mitochondrial neurogastrointestinal encephalomyopathy family. Open symbols indicate unaffected individuals and filled symbols indicate an affected individual. Half-filled symbols indicate individuals with one heterozygous mutation. The arrow indicates the proband. Genotypes of both TYMP mutations are indicated underneath each examined individual. TYMP, thymidine phosphorylase gene.

$R R M 2 B$ and $T K 2$ genes were amplified using PCR. The primer sequences and PCR conditions used are available on request. PCR products were sequenced by an automatic genetic analyzer ABI 3130xl using a BigDye terminator cycle sequencing ready reaction kit (Applied Biosystems). Sequence variations were identified by the SeqScape (ver. 2.1, Applied Biosystems) and Chromas software (ver. 2.33, Technelysium, South Brisbane, Australia). Sequence variations were confirmed by analyzing both strands of DNA. cDNA numbering was achieved with +1 corresponding to the A of the ATG initiation codon, according to the mutation nomenclature instruction of the Human Genome Variation Society (http://www.hgvs.org/mutnomen/). In silico predictions were performedusing theSIFT(http://sift.jcvi.org/), PolyPhen-2 (http://genetics.bwh.harvard.edu/pph2/) and MUpro (SVM; http://mupro.proteomics.ics.uci.edu/) programs. Multiple alignments of amino acid sequences among different species were performed using the MEGA5 program (ver 5.05) (22). Deletion of 4977 bp (m.8470_13446del4977) in mtDNA, frequently called a 'common deletion', was detected using an Expand Long Template PCR System (Roche, Mannheim, Germany). The used PCR primers covered the following regions of the revised Cambridge reference sequence: 8225-8247 (forward) and 13707-13729 (reverse).

\section{Results}

Identification of compound heterozygous mutations in TYMP. Mutation screening revealed two TYMP heterozygous missense mutations; Thr151Pro (c.451A $>$ C) and Leu270Pro (c.809T>C; Fig. 2A). One of these mutations was transmitted from each parent of the patient; Leu270Pro from the father and Thr151Pro from the mother. The patient had two unaffected siblings, each receiving a single mutation from their parents. These mutations were not reported in the dbSNP137 (http://ncbi.nlm.nih.gov/) and 1000 Genome Database (http://www.1000genomes.org/), and were not found in the 225 controls. Both mutation sites were highly conserved 
Table I. Compound heterozygous mutations of TYMP in a MNGIE family.

\begin{tabular}{|c|c|c|c|c|c|c|}
\hline \multirow[b]{2}{*}{ Family } & \multicolumn{2}{|c|}{ Substitution } & \multirow[b]{2}{*}{ Amino acid } & \multirow[b]{2}{*}{ Phenotype } & \multirow[b]{2}{*}{ Inheritance } & \multirow{2}{*}{$\begin{array}{l}\text { In silico analysis } \\
(\mathrm{S} / \mathrm{P} 2 / \mathrm{M})^{\mathrm{b}}\end{array}$} \\
\hline & Gene & Nucleotide ${ }^{a}$ & & & & \\
\hline \multirow[t]{2}{*}{ MT119 } & TYMP & c. $451 \mathrm{~A}>\mathrm{C}$ & $\mathrm{T} 151 \mathrm{P}$ & MNGIE & Recessive & $0.00^{c} / 1.00^{c} / 1$ \\
\hline & & c.809T>C & L270P & & & $0.00^{\mathrm{c}} / 1.00^{\mathrm{c}} /-^{\mathrm{c}}$ \\
\hline
\end{tabular}

Table II. Variants identified from whole mtDNA in the MNGIE patient (III-2).

\begin{tabular}{|c|c|c|c|c|c|}
\hline Gene & Nt position ${ }^{a}$ & Nucleotide change & Amino acid change & Characterization & Report \\
\hline \multirow[t]{7}{*}{ D-loop } & 73 & $A>G$ & - & Poly & Yes \\
\hline & 152 & $\mathrm{~T}>\mathrm{C}$ & - & Poly & Yes \\
\hline & 200 & $\mathrm{~A}>\mathrm{G}$ & - & Poly & Yes \\
\hline & 263 & $\mathrm{~A}>\mathrm{G}$ & - & Poly & Yes \\
\hline & 315.1 & $\mathrm{C}$ insertion & - & Poly & Yes \\
\hline & 523 & A deletion & - & Poly & Yes $^{b}$ \\
\hline & 524 & $\mathrm{C}$ deletion & - & Poly & $\mathrm{Yes}^{\mathrm{b}}$ \\
\hline \multirow[t]{3}{*}{$12 S r R N A$} & 663 & $\mathrm{~A}>\mathrm{G}$ & - & Poly & Yes \\
\hline & 750 & $\mathrm{~A}>\mathrm{G}$ & - & Poly & Yes \\
\hline & 1438 & $\mathrm{~A}>\mathrm{G}$ & - & Poly & Yes \\
\hline \multirow[t]{2}{*}{$16 S r R N A$} & 1736 & $\mathrm{~A}>\mathrm{G}$ & - & Poly & Yes \\
\hline & 2706 & $\mathrm{~A}>\mathrm{G}$ & - & Poly & Yes \\
\hline ND1 & 4248 & $\mathrm{~T}>\mathrm{C}$ & Syn & Poly & Yes \\
\hline \multirow[t]{2}{*}{ ND2 } & 4769 & $\mathrm{~A}>\mathrm{G}$ & Syn & Poly & Yes \\
\hline & 4824 & $\mathrm{~A}>\mathrm{G}$ & Thr119Ala & Poly & Yes \\
\hline \multirow[t]{2}{*}{ COI } & 6060 & $\mathrm{~A}>\mathrm{G}$ & Ile53Val & Poly & Yes \\
\hline & 7028 & $\mathrm{C}>\mathrm{T}$ & Syn & Poly & Yes \\
\hline \multirow[t]{2}{*}{ ATP6 } & 8794 & $\mathrm{C}>\mathrm{T}$ & His90Tyr & Poly/exercise endurance & Yes \\
\hline & 8860 & $\mathrm{~A}>\mathrm{G}$ & Thr112Ala & Poly & Yes \\
\hline ND4 & 11719 & $\mathrm{G}>\mathrm{A}$ & Syn & Poly & Yes \\
\hline ND5 & 12705 & $\mathrm{C}>\mathrm{T}$ & Syn & Poly & Yes \\
\hline \multirow[t]{2}{*}{$C y t B$} & 14766 & $\mathrm{C}>\mathrm{T}$ & Thr7Ile & Poly & Yes \\
\hline & 15326 & $\mathrm{~A}>\mathrm{G}$ & Thr194Ala & Poly & Yes \\
\hline \multirow[t]{4}{*}{ D-loop } & 16223 & $\mathrm{C}>\mathrm{T}$ & - & Poly & Yes \\
\hline & 16290 & $\mathrm{C}>\mathrm{T}$ & - & Poly & Yes \\
\hline & 16319 & $\mathrm{G}>\mathrm{A}$ & - & Poly & Yes \\
\hline & 16362 & $\mathrm{~T}>\mathrm{C}$ & - & Poly & Yes \\
\hline
\end{tabular}

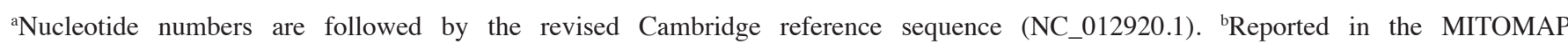
(http://mitomap.org/MITOMAP), but not in the mtDB (http://www.mtdb.igp.uu.se). Syn, synonymous mutation; MNGIE, mitochondrial neurogastrointestinal encephalomyopathy.

among different species (Fig. 2B). Several in silico analyses predicted that the mutations affect protein function (Table I). No other causative mutation was observed in the examined nuclear genes.
Identification of mtSNPs. Whole mtDNA sequencing revealed numerous mitochondrial single nucleotide polymorphisms (mtSNPs; Table II). However, all the mtSNPs have been reported to be polymorphic in the MITOMAP-Human Mitochondrial 
A

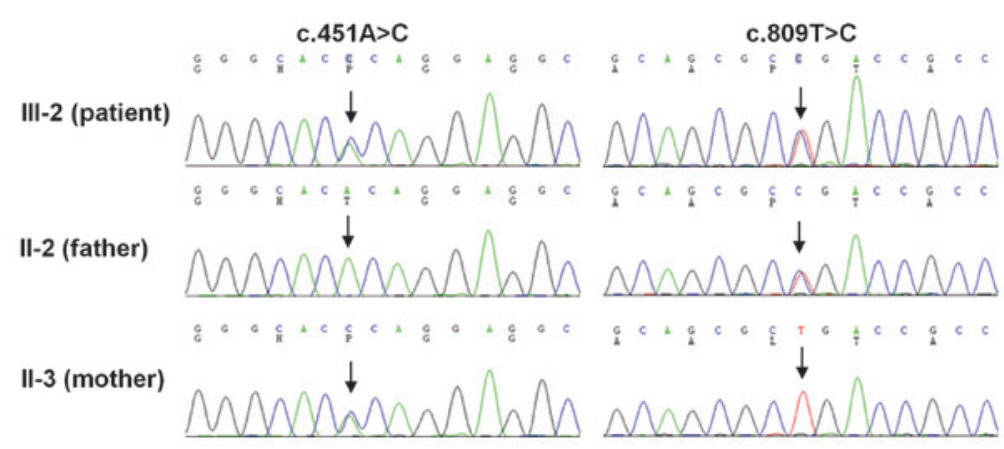

B



Figure 3. Brain MRI of the MNGIE patient. (A) Axial T2-weighted image (B) Coronal T2-weighted image. Brain T2-weighed MRI showed diffuse cerebral white matter hyperintensity with bilateral thalamic involvement. MNGIE, mitochondrial neurogastrointestinal encephalomyopathy.

Genome Database (http://mitomap.org/MITOMAP) or the mtDB-Human Mitochondrial Genome Database (http://www.mtdb.igp.uu.se) (24). Although ATP6
m.8794C $>\mathrm{T}$ (His90Tyr) has been associated with high-performance endurance running (25), it was not considered to be causative for MNGIE, as it was observed in the controls and has also been reported to be a polymorphic mtSNP in the MITOMAP. The long template PCR from the blood DNA of the patient revealed no common large deletion of mtDNA (m.8470_13446del4977).

Clinical manifestations. A 28-year-old female patient was referred to Kangbuk Samsung Hospital (Seoul, Korea) due to abdominal pain, diarrhea, fever $\left(\leq 39.5^{\circ} \mathrm{C}\right)$ and headaches. Computed tomography (CT) scans revealed a diffuse white matter change in the brain. In early childhood, the patient frequently suffered from abdominal pain, diarrhea and vomiting approximately once a week, which was aggravated by febrile illness. Since elementary school, the patient's capacity for exercise has been decreased. In high school, the patient had cosmetic surgery to correct bilateral ptosis. At 21 years old the patient had an eye operation to correct strabismus, but did not suffer from diplopia. One year ago, the patient was diagnosed with fatty liver and polycystic ovary syndrome. Upon admission, the patient's vital signs were stable with a BMI of $18 \mathrm{~kg} / \mathrm{m}^{2}$ (height $165 \mathrm{~cm}$ and weight $49 \mathrm{~kg}$ ). The patient did not appear to exhibit cachexia.

Neurological examination revealed mild bilateral ptosis, mild bilateral external ophthalmoparesis and areflexia of stretch reflexes; however, there was no cognitive dysfunction or motor or sensory abnormalities. Pigmentary retinopathy was observed in both eyes upon fundus examination. Nerve conduction study revealed demyelinating type diffuse sensory motor polyneuropathy and there were conduction blocks on bilateral tibial nerves. Needle EMG revealed normal insertional and spontaneous activity, and neurogenic motor unit potential without 
myogenic potential. Ultrasonographic study of the peripheral nerves in all four extremities revealed mild nerve enlargement of bilateral median nerves. Somatosensory evoked potential studies revealed peripheral conduction defects. A visual evoked potential study demonstrated conduction delay. An MRI revealed diffuse white matter high signal intensity with slight involvement of the deep nuclei, brainstem and cerebellum; however, MR spectroscopy did not reveal any abnormalities (Fig. 3).

In the laboratory study, the level of creatine kinase was $1240 \mathrm{IU} / 1$, serum lactic acid was $1.98 \mathrm{mmol} / \mathrm{l}$ (normal, $0.5-2.2$ ) and pyruvic acid was $0.038 \mathrm{mmol} / 1$ (normal, 0.034-0.102), and the cerebrospinal fluid study was normal. Examinations for leukodystrophy (arylsulfatase A, N-acetyl aspartic acid, $\beta$-galactocerebrosidase and phytanic acid) revealed normal levels. Buffy coat TP activity decreased to $9.6 \%$ of the normal level (patient, $61 \mathrm{nmol} / \mathrm{h} / \mathrm{mg}$ protein; normal, $634 \pm 217 \mathrm{nmol} / \mathrm{h} / \mathrm{mg}$ protein).

\section{Discussion}

The present study reports an autosomal recessive early-onset but mild phenotype MNGIE patient with compound heterozygous TYMP mutations Thr151Pro (c.451A $>$ C) and Leu270Pro (c.809T $>$ C). Since i) these mutations were not found in healthy controls and no unaffected familial member had both mutations; ii) the mutation sites were well conserved; iii) both mutations caused an amino acid substitution with Pro residue which frequently acts as a structural disruptor of protein secondary structure such as alpha helix; and iv) no other causative mutations were identified in the nuclear genes or the mtDNA, we hypothesize that the two identified heterozygous mutations may be the underlying cause of MNGIE.

The TYMP gene contains 10 exons spanning $>4.3 \mathrm{~kb}(25)$ and encodes TP, which catalyzes the phosphorolysis of thymidine, an essential step in the nucleotide salvage pathway for mtDNA replication (4). The 482-residue TP has a molecular mass of $49.97 \mathrm{kD}$ and usually occurs as a homodimeric structure (26).

To date, several homozygous and compound heterozygous TYMP mutations have been reported to be causative of autosomal recessive MNGIE (5,12-17). Homozygous mutations in TYMP usually result in an almost complete abolition of TP activity, with $<5 \%$ remaining $(5,13,14)$; however, compound heterozygous mutations exhibit a partial loss of activity with $10-20 \%$ normal TP activity $(15,16)$. Although heterozygous carriers are clinically asymptomatic, TP activity is decreased to $26-35 \%$ of the normal level (15). Patients with compound heterozygous mutations have been reported to have mild symptoms with a later onset than that found in typical homozygous patients $(15,16)$. In an Italian family, compound heterozygous mutations in TYMP exhibited different clinical presentations between two affected siblings (17).

With the exception of cachexia, the patient suffered from the majority of symptoms associated with MNGIE, including frequent GI discomfort, external ophthalmoplegia, pigmentary retinopathy and demyelinating type diffuse sensory motor polyneuropathy. The TP activity of the patient was determined to be $9.6 \%$ of the normal value, which corresponds to an intermediate level between that of the mild phenotype of late-onset heterozygous patients and that of the severe cachexic phenotype of homozygous patients $(5,13)$.

To the best of our knowledge, this is the first report of a Korean MNGIE patient with compound heterozygous mutations in TYMP. The patient revealed an early-onset but mild phenotype; therefore, patients with heterozygous mutations may exhibit mild phenotypes with variable onset ages, partially based on the reduction in levels of TP activity.

\section{Acknowledgements}

This study was supported by a grant from the Korean Health Technology R\&D Project, Ministry of Health \& Welfare (A120814) and the National Research Foundation (NRF) grant, funded by the Ministry of Education, Science and Technology, Republic of Korea (2011-0013694).

\section{References}

1. Spelbrink JN, Li FY, Tiranti V, et al: Human mitochondrial DNA deletions associated with mutations in the gene encoding Twinkle, a phage T7 gene 4-like protein localized in mitochondria. Nat Genet 28: 223-231, 2001.

2. Hirano M, Nishigaki $Y$ and Martí R: Mitochondrial neurogastrointestinal encephalomyopathy (MNGIE): a disease of two genomes. Neurologist 10: 8-17, 2004.

3. Spinazzola A and Zeviani M: Disorders of nuclear-mitochondrial intergenomic signaling. Gene 354: 162-168, 2005.

4. Suomalainen A and Isohanni P: Mitochondrial DNA depletion syndromes - many genes, common mechanisms. Neuromuscul Disord 20: 429-437, 2010.

5. Nishino I, Spinazzola A and Hirano M: Thymidine phosphorylase gene mutations in MNGIE, a human mitochondrial disorder. Science 283: 689-692, 1999.

6. Vissing J, Ravn K, Danielsen ER, et al: Multiple mtDNA deletions with features of MNGIE. Neurology 59: 926-929, 2002.

7. Van Goethem G, Schwartz M, Löfgren A, et al: Novel POLG mutations in progressive external ophthalmoplegia mimicking mitochondrial neurogastrointestinal encephalomyopathy. Eur J Hum Genet 11: 547-549, 2003.

8. Shaibani A, Shchelochkov OA, Zhang S, et al: Mitochondrial neurogastrointestinal encephalopathy due to mutations in RRM2B. Arch Neurol 66: 1028-1032, 2009.

9. Kollberg G, Darin N, Benan K, et al: A novel homozygous RRM2B missense mutation in association with severe mtDNA depletion. Neuromuscul Disord 19: 147-150, 2009.

10. Naviaux RK and Nguyen KV: POLG mutations associated with Alpers' syndrome and mitochondrial DNA depletion. Ann Neurol 55: 706-712, 2004.

11. Tyynismaa H, Ylikallio E, Patel M, Molnar MJ, Haller RG and Suomalainen A: A heterozygous truncating mutation in RRM2B causes autosomal-dominant progressive external ophthalmoplegia with multiple mtDNA deletions. Am J Hum Genet 85: 290-295, 2009.

12. Gamez J, Ferreiro C, Accarino ML, et al: Phenotypic variability in a Spanish family with MNGIE. Neurology 59: 455-457, 2002

13. Szigeti K, Wong LJ, Perng CL, et al: MNGIE with lack of skeletal muscle involvement and a novel TP splice site mutation. J Med Genet 41: 125-129, 2004.

14. Hirano M, Lagier-Tourenne C, Valentino ML, Martí R and Nishigaki Y: Thymidine phosphorylase mutations cause instability of mitochondrial DNA. Gene 354: 152-156, 2005.

15. Martí R, Verschuuren JJ, Buchman A, et al: Late-onset MNGIE due to partial loss of thymidine phosphorylase activity. Ann Neurol 58: 649-652, 2005.

16. Massa R, Tessa A, Margollicci M, et al: Late-onset MNGIE without peripheral neuropathy due to incomplete loss of thymidine phosphorylase activity. Neuromuscul Disord 19: 837-840, 2009.

17. Libernini L, Lupis C, Mastrangelo M, et al: Mitochondrial neurogastrointestinal encephalomyopathy: novel pathogenic mutations in thymidine phosphorylase gene in two Italian brothers. Neuropediatrics 43: 201-208, 2012. 
18. Ishikawa F, Miyazono K, Hellman U, et al: Identification of angiogenic activity and the cloning and expression of platelet-derived endothelial cell growth factor. Nature 338: 557-562, 1989.

19. Bakker JA, Schlesser P, Smeets HJ, Francois B and Bierau J: Biochemical abnormalities in a patient with thymidine phosphorylase deficiency with fatal outcome. J Inherit Metab Dis 2010: Feb 12, 2010 (Epub ahead of print).

20. Haraguchi M, Tsujimoto H, Fukushima M, et al: Targeted deletion of both thymidine phosphorylase and uridine phosphorylase and consequent disorders in mice. Mol Cell Biol 22: 5212-5222, 2002.

21. López LC, Akman HO, García-Cazorla A, et al: Unbalanced deoxynucleotide pools cause mitochondrial DNA instability in thymidine phosphorylase-deficient mice. Hum Mol Genet 18: 714-722, 2009.

22. Tamura K, Peterson D, Peterson N, Stecher G, Nei M and Kumar S: MEGA5: molecular evolutionary genetics analysis using maximum likelihood, evolutionary distance, and maximum parsimony methods. Mol Biol Evol 28: 2731-2739, 2011.
23. Ingman $M$ and Gyllensten U: mtDB: Human Mitochondrial Genome Database, a resource for population genetics and medical sciences. Nucleic Acids Res 34: D749-D751, 2006.

24. Tanaka M, Takeyasu T, Fuku N, Li-Jun G and Kurata M: Mitochondrial genome single nucleotide polymorphisms and their phenotypes in the Japanese. Ann NY Acad Sci 1011: 7-20, 2004.

25. Hagiwara K, Stenman G, Honda H, et al: Organization and chromosomal localization of the human platelet-derived endothelial cell growth factor gene. Mol Cell Biol 11: 2125-2132, 1991.

26. Asai K, Nakanishi K, Isobe I, et al: Neurotrophic action of gliostatin on cortical neurons. Identity of gliostatin and platelet-derived endothelial cell growth factor. J Biol Chem 267: 20311-20316, 1992. 